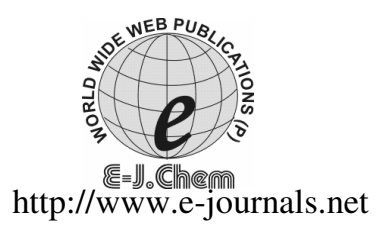

ISSN: 0973-4945; CODEN ECJHAO

E-Journal of Chemistry 2009, 6(S1), S53-S58

\title{
Synthesis of Multi-substituted 4,5-Dihydrofuran Derivatives from $(S)$-Limonene and 1,3-Dicarbonyl Compounds and their Biological Activities
}

\author{
E. FINDIK*, A. DİNGİL, I. KARAMAN ${ }^{\S}$, Y. BUDAK and M. CEYLAN \\ Department of Chemistry, Faculty of Arts and Sciences, \\ Gaziosmanpasa University-60250, Tokat, Turkey. \\ ${ }^{\S}$ Department of Biology, Faculty of Arts and Sciences, \\ Gaziosmanpasa University-60250, Tokat, Turkey. \\ esrafndk@gmail.com
}

Received 23 December 2008; Accepted 20 February 2009

\begin{abstract}
Multi-substituted 4,5-dihydrofuran derivatives were regioselectively synthesized by the reaction of $\alpha$-carbo radical produced from 1,3-dicarbonyl compounds by oxidation with $\mathrm{Mn}(\mathrm{OAc})_{3}$ in Acetic acid and $S$-(-)-limonene. All the compounds were tested for their antibacterial and antifungal activities by the disc-diffusion technique.
\end{abstract}

Keywords: Synthesis, Limonene, Biological activities.

\section{Introduction}

Monoterpenes are widely used in the pharmaceutical, cosmetic and food industries as active components of drugs and ingredients of artificial flavours and fragrances ${ }^{1,2}$. Monoterpenes and limonene in particular, are an important class of naturally occurring chiral compounds ${ }^{3}$ widely used in organic synthesis ${ }^{4}$ as starting materials for optically pure molecules ${ }^{5}$, as a core of chiral auxiliaries $^{6}$ and as asymmetric ligands employed in enantioselective transformations ${ }^{7}$. In nature, limonene is abundant in both enantiomeric forms, $(R)$-limonene and $(S)$-limonene ${ }^{8}$. From a chemical point of view, these terpenes are very versatile since they present two double bonds that can be selectively converted to several functional groups ${ }^{9-11}$. The biological activities of $(R)$-limonene and $(S)$-limonene have been studied ${ }^{12}$ against Staphylococcus aureus ATCC 12600, Bacillus cereus ATCC 11778, Enterococcus faecalis ATCC 29212, Escherichia coli ATCC 11775, Pseudomonas aeruginosa ATCC 9027, Klebsiella pneumoniae ATCC 13883 and Cryptococcus neoformans ATCC 90112 bacteria. They have shown considerable activity.

In addition, furans and their derivatives are available in nature ${ }^{3}$ and one of the most important heterocyclic compounds ${ }^{14}$ for the total synthesis of the complicated naturally occurring metabolites in organic chemistry ${ }^{15}$. Furans and their derivatives have a broad spectrum of biological activity such as antiprotozal ${ }^{16}$, antiviral ${ }^{17}$, antimicrobial ${ }^{17}$, anticancer ${ }^{17}$, HIV-1 $_{\text {activity }}{ }^{18}$ and antimalarial ${ }^{19}$. 
Therefore, this study focuses on the synthesis of 4,5-dihydrofuran derivatives $\mathbf{3}$ and 4 with a limonene and dihydrofuran ring and the determination of their biological activities toward 12 different human pathogen microorganisms.

\section{Experimental}

$\mathrm{Mn}(\mathrm{OAc})_{3},(S)$-limonene, Acetic acid and 1,3-dicarbonyls were commercial products with highest reagent grade. IR spectra (neat or in $\mathrm{CHCl}_{3}$ ) were recorded on a Jasco FT/IR-430 spectrometer. ${ }^{1} \mathrm{H}$ and ${ }^{13} \mathrm{C}$ NMR spectra were recorded on a Bruker Avance III 400 instrument. As internal standards served TMS $(\delta 0.00)$ for ${ }^{1} \mathrm{H}$ NMR and $\mathrm{CDCl}_{3}(\delta 77.0)$ for ${ }^{13} \mathrm{C}$ NMR spectroscopy; $J$ values are given in $H z$. The multiplicities of the signals in the ${ }^{1} \mathrm{H}$ NMR spectra are abbreviated by s (singlet), d (doublet), $t$ (triplet), q (quartet), m (multiplet), br (broad) and combinations thereof. Elemental analyses were obtained by a LECO CHNS 932 Elemental Analyzer.

\section{General procedure for the synthesis of benzofuran and 4,5-dihydrofuran derivatives}

A solution of $\mathrm{Mn}(\mathrm{OAc})_{3}(2.97 \mathrm{~g}, 11.1 \mathrm{mmol})$ in $15 \mathrm{~mL}$ of glacial Acetic acid was heated under $\mathrm{N}_{2}$ atmosphere at $80{ }^{\circ} \mathrm{C}$ until it dissolved. After $\mathrm{Mn}(\mathrm{OAc})_{3}$ was dissolved completely, the solution was cooled to $60{ }^{\circ} \mathrm{C}$ and a solution of 1,3 dicarbonyl compounds variable $(1.04 \mathrm{~g}$, $7.4 \mathrm{mmol})$ and $S$-(-)-limonene $(0.5 \mathrm{~g}, 3.7 \mathrm{mmol})$ in $5 \mathrm{~mL}$ acetic acid was added to this mixture. The reaction was finished when the dark brown colour of the solution disappeared. Acetic acid was evaporated under reduced pressure; $\mathrm{H}_{2} \mathrm{O}$ was added to the residue and extracted with ethyl acetate $(3 \times 20 \mathrm{~mL})$. The combined organic phases were neutralized with saturated $\mathrm{NaHCO}_{3}$ solution, dried $\left(\mathrm{Na}_{2} \mathrm{SO}_{4}\right)$, and evaporated. Crude products were purified by column chromatography on silica gel using $n$-hexane/ethyl acetate $(9: 1)$ as eluent.

Mixture of (R)-2,6,6-trimethyl-2-((S)-4-methylcyclohex-3-enyl)-2,3,6,7-tetrahydrobenzofuran-4(5H)-one (3a) and (S)-2,6,6-trimethyl-2-((S)-4-methylcyclohex-3-enyl)2,3,6,7-tetrahydrobenzofuran-4(5H)-one (4a): Viscous oil; ${ }^{1} \mathbf{H}$ NMR (400 $\left.\mathrm{MHz}, \mathrm{CDCl}_{3}\right)$ : $\delta=5.24(\mathrm{bs}, 1 \mathrm{H}), 2.64(\mathrm{~d}, J=14.3 \mathrm{~Hz}, 1 \mathrm{H}), 2.31(\mathrm{t}, J=13.7 \mathrm{~Hz}, 1 \mathrm{H}), 2.17-1.08(\mathrm{~m}, 4 \mathrm{H})$, 1.92-1.86 (m, 3H), $1.53(\mathrm{~s}, 3 \mathrm{H}), 1.24(\mathrm{~d}, J=4.7 \mathrm{~Hz}, 3 \mathrm{H}), 1.18-1.10(\mathrm{~m}, 1 \mathrm{H}), 1.05(\mathrm{~s}, 3 \mathrm{H})$, 1.03 (s, 3H). ${ }^{13} \mathbf{C}$ NMR (100 MHz, $\left.\mathrm{CDCl}_{3}\right): \delta=194.57,(175.20-175.03),(133.84-133.70)$, (119.79-119.73), 110.98, (95.39-95.17), 50.72, (43.03-42.84), 37.82, 35.01, (34.27-33.95), (30.31-30.21), (28.87-28.84), (28.33-28.28), (26.19-25.88), (24.78-24.45), 23.33, (23.2723.17). IR (Liquid): 3046, 3010, 2960, 2928, 2890, 1633, 1402, 1240, 1028, $759 \mathrm{~cm}^{-1}$. Anal. Calcd for $\mathrm{C}_{18} \mathrm{H}_{26} \mathrm{O}_{2}$ : C, 78.79; H, 9.55; Found: C, 78.47; H, 9.58.

Mixture of (R)-2-methyl-2-((S)-4-methylcyclohex-3-enyl)-2,3,6,7-tetrahydrobenzofuran-4(5H)-one (3b) and (S)-2-methyl-2-((S)-4-methylcyclohex-3-enyl)2,3,6,7-tetrahydrobenzofuran-4(5H)-one (4b): Viscous oil, ${ }^{\mathbf{1}} \mathbf{H}$ NMR (400 MHz, $\left.\mathrm{CDCl}_{3}\right): \delta=5.29(\mathrm{bs}, 1 \mathrm{H}), 2.69(\mathrm{~d}, J=14.3 \mathrm{~Hz}, 1 \mathrm{H}), 2.39-2.34(\mathrm{~m}, 3 \mathrm{H}), 2.27-2.26(\mathrm{~m}, 2 \mathrm{H})$, 1.98-1.87 (m, 5H), 1.74-1.66 (m, 2H), 1.59-1.57 (m, 4H), 1.29-1.26 (m, 4H). ${ }^{13}$ C NMR (100 $\left.\mathrm{MHz}, \mathrm{CDCl}_{3}\right): \delta=(176.43-176.29),(133.98-133.85),(119.79-119.75), 112.63$, (95.2895.08), (43.10-42.94), 36.31, 35.27, 34.63, (30.34-30.28), (26.25-25.97), 24.51, 24.18, 23.97, (23.40-23.31), 21.70. IR (Liquid): 3009, 2927, 2889, 2839, 1632, 1402, 1265, 999, 856 $\mathrm{cm}^{-1}$. Anal. Calcd for $\mathrm{C}_{16} \mathrm{H}_{22} \mathrm{O}_{2}$ : C, 78.01; H, 9.00; Found: C, 77.81; H, 8.77.

Mixture of 1-((R)-5-methyl-5-((S)-4-methylcyclohex-3-enyl)-2-phenyl-4,5-dihydrofuran-3-yl) ethanone (3c) and 1-((S)-5-methyl-5-((S)-4-methylcyclohex-3-enyl)-2-phenyl-4,5dihydrofuran-3-yl)ethanone (4c): Viscous oil; ${ }^{1} \mathbf{H}$ NMR $\left(400 \mathrm{MHz}, \mathrm{CDCl}_{3}\right): \delta=7.51$ $7.49(\mathrm{~m}, 2 \mathrm{H}), 7.46-7.41(\mathrm{~m}, 3 \mathrm{H}), 5.37$ (bs, $1 \mathrm{H}), 3.06(\mathrm{~d}, J=14.8 \mathrm{~Hz}, 1 \mathrm{H}), 2.72$ (dd, $J=14.8$, 
$10 \mathrm{~Hz}, 1 \mathrm{H}), 2.15-1.93(\mathrm{~m}, 5 \mathrm{H}), 1.90(\mathrm{~s}, 3 \mathrm{H}), 1.89-1.78(\mathrm{~m}, 5 \mathrm{H}), 1.64(\mathrm{~s}, 3 \mathrm{H}), 1.40(\mathrm{~s}$, $3 \mathrm{H}) .{ }^{13} \mathrm{C}$ NMR $\left(100 \mathrm{MHz}, \mathrm{CDCl}_{3}\right): \delta=194.80,(165.73-165.65),(134.06-133.93), 131.49$, 130.36, (129.02-128.98), (128.33-128.29), (119.96-119.93), 114.57, (91.04-90.81), (43.2442.96), 40.49, 39.75, (30.47-30.37), 28.76, 26.39, 26.07, 24.14, 23.54, 23.37. IR (Liquid): 3059, 3008, 2965, 2926, 2836, 1623, 1591, 1376, 1267, 1243, 1072, 1062, 786,761 cm $\mathrm{cm}^{-1}$ Anal. Calcd for $\mathrm{C}_{20} \mathrm{H}_{24} \mathrm{O}_{2}$ : C, 81.04; H, 8.16; Found: C, 79.86; H, 7.96.

Mixture of (R)-methyl 2,5-dimethyl-5-(S)-4-methylcyclohex-3-enyl)-4,5-dihydrofuran-3carboxylate (3d) and (S)-methyl 2,5-dimethyl-5-((S)-4-methylcyclohex-3-enyl)-4,5dihydrofuran-3-carboxylate (4d): Viscous oil; ${ }^{1} \mathbf{H}$ NMR $\left(400 \mathrm{MHz}, \mathrm{CDCl}_{3}\right): \delta=5.32$ (bs, $1 \mathrm{H}), 3.64(\mathrm{~s}, 3 \mathrm{H}), 2.76(\mathrm{~d}, J=14.4 \mathrm{~Hz}, 1 \mathrm{H}), 2.44-2.38(\mathrm{dd}, J=12.4,10.8 \mathrm{~Hz}, 1 \mathrm{H})$, $2.12(\mathrm{~s}, 3 \mathrm{H}), 1.95-1.87(\mathrm{~m}, 3 \mathrm{H}), 1.78-1.62(\mathrm{~m}, 3 \mathrm{H}), 1.59(\mathrm{~s}, 3 \mathrm{H}), 1.26-1.22(\mathrm{~m}, 4 \mathrm{H})$. ${ }^{13}$ C NMR (100 MHz, $\left.\mathrm{CDCl}_{3}\right): \delta=(167.26-167.11),(13.95-133.77),(120.01-119.92)$, 100.68, (90.62-90.40), 50.58, (43.10-42.92), 38.93, 38.16, (30.42-30.32), (26.22-25.95), 24.60, 24.15, (23.29-23.26), 14.13. IR $\left(\mathrm{CHCl}_{3}\right): 3010,2947,2925,2840,1702,1644$, 1436, 1381, 1241, 1097, $762 \mathrm{~cm}^{-1}$. Anal. Calcd for $\mathrm{C}_{15} \mathrm{H}_{22} \mathrm{O}_{3}: \mathrm{C}, 71.97 ; \mathrm{H}, 8.86$; Found: C, 71.97; H, 8.86.

Mixture of (R)-ethyl 2,5-dimethyl-5-((S)-4-methylcyclohex-3-enyl)-4,5-di-hydrofuran3-carboxylate (3e) and (S)-ethyl 2,5-dimethyl-5-((S)-4-methylcyclohex-3-enyl)-4,5dihydrofuran-3-carboxylate (4e): Viscous oil; ${ }^{1} \mathbf{H}$ NMR $\left(400 \mathrm{MHz}, \mathrm{CDCl}_{3}\right): \delta=5.25$ (bs, $1 \mathrm{H}), 4.04(\mathrm{q}, J=8 \mathrm{~Hz}, 2 \mathrm{H}), 2.71(\mathrm{~d}, J=14 \mathrm{~Hz}, 1 \mathrm{H}), 2.39-2.33(\mathrm{dd}, J=13.6,9 \mathrm{~Hz}, 1 \mathrm{H})$, $2.06(\mathrm{~s}, 3 \mathrm{H}), 1.90-1.82(\mathrm{~m}, 3 \mathrm{H}), 1.72-1.59(\mathrm{~m}, 3 \mathrm{H}), 1.53(\mathrm{~s}, 3 \mathrm{H}), 1.19-1.15(\mathrm{~m}, 7 \mathrm{H})$. ${ }^{13}$ C NMR (100 MHz, $\left.\mathrm{CDCl}_{3}\right): \delta=(169.79-166.65),(133.77-133.60),(120.01-119.91)$, 100.86, (90.34-90.12), 59.10, (43.06-42.88), 38.94, 38.20, (30.37-30.28), 26.18, 25.91, 24.04, 23.34, 23.22, (14.36-14.06). IR $\left(\mathrm{CHCl}_{3}\right): 2968,2927,2727,1698,1646,1445$, 1380, 1240, 1220, 974, 785, $762 \mathrm{~cm}^{-1}$. Anal. Calcd for $\mathrm{C}_{16} \mathrm{H}_{24} \mathrm{O}_{3}: \mathrm{C}, 72.69 ; \mathrm{H}, 9.15$; Found: C, 72.96; H, 8.84.

Mixture of (R)- $N, N$-diethyl-2,5-dimethyl-5-((S)-4-methylcyclohex-3-enyl)-4,5-dihydrofuran-3-carboxamide (3f) and (S)- $N, N$-diethyl-2,5-dimethyl-5-((S)-4-methylcyclohex3-enyl)-4,5-dihydrofuran-3-carboxamide (4f): Viscous oil; ${ }^{1} \mathbf{H}$ NMR $\left(400 \mathrm{MHz}, \mathrm{CDCl}_{3}\right): \delta=$ $5.32(\mathrm{bs}, 1 \mathrm{H}), 3.33(\mathrm{q}, J=7.2 \mathrm{~Hz}, 4 \mathrm{H}), 2.81$ (ddd, $J=14.2,5.6,1.6 \mathrm{~Hz}, 1 \mathrm{H}), 2.41$ (d, $J=$ $14.4 \mathrm{~Hz}, 1 \mathrm{H}), 2.02-1.88(\mathrm{~m}, 3 \mathrm{H}), 1.79(\mathrm{~d}, J=1.6 \mathrm{~Hz}, 3 \mathrm{H}), 1.74-1.64(\mathrm{~m}, 2 \mathrm{H}), 1.59(\mathrm{~s}, 3 \mathrm{H})$, 1.39-1.37 (m, 1H), $1.25(\mathrm{~s}, 3 \mathrm{H}), 1.25-1.18(\mathrm{~m}, 1 \mathrm{H}), 1.09(\mathrm{t}, J=7.2 \mathrm{~Hz}, 6 \mathrm{H}) .{ }^{13} \mathbf{C}$ NMR $(100$ $\left.\mathrm{MHz}, \mathrm{CDCl}_{3}\right): \delta=168.21,(155.26-155.26),(134.02-133.82),(120.14-120.07),(103.36-$ 103.32), (88.85-88.69), (43.11-42.97), 41.80, 40.83, (30.48-30.44), 26.40, 26.17, 24.67, 24.04, 23.57, 23.34, 13.78, (13.45-13.42). IR (Liquid): 3425, 2968, 2929, 2835, 1636, 1433 , 1358, 1134, $785 \mathrm{~cm}^{-1}$. Anal. Calcd for $\mathrm{C}_{18} \mathrm{H}_{29} \mathrm{NO}_{2}$ : C, 74.18; H, 10.03; N, 4.81; Found: C, $67.18 ; \mathrm{H}, 9.92 ; \mathrm{N}, 4.42$.

Mixture of 1-((R)-2,5-dimethyl-5-((S)-4-methylcyclohex-3-enyl)-4,5-dihydrofuran-3yl)ethanone (3g) and 1-(S)-2,5-dimethyl-5-((S)-4-methylcyclohex-3-enyl)-4,5-dihydrofuran3-yl)ethanone (4g): Viscous oil; ${ }^{1} \mathbf{H}$ NMR $\left(400 \mathrm{MHz}, \mathrm{CDCl}_{3}\right): \delta=5.30$ (bs, $\left.1 \mathrm{H}\right), 2.80(\mathrm{~d}, J=$ $14 \mathrm{~Hz}, 1 \mathrm{H}), 2.45$ (dd, $J=13.4,8.4 \mathrm{~Hz}, 1 \mathrm{H}), 2.12$ (bs, 3H), 2.11 (bs, 3H), 1.98-1.90 (m, 4H), 1.75- 1.59 (m, 3H), 1.57 (s, 3H), 1.23 (bs, 3H). ${ }^{13} \mathbf{C ~ N M R ~}\left(100 \mathrm{MHz}, \mathrm{CDCl}_{3}\right): \delta=194.68$, (167.03-166.88), (133.95-133.80), (119.89-119.83), 111.66, (90.78-90.58), (43.06-42.89), (39.69-39.96), (30.37-30.28), 29.22, (26.20-25.95), 24.56, 24.13, (23.37-23.28), 15.17. IR (Liquid): 3010, 2926, 1698, 1653, 1600, 1436, 1378, 1244, 937, 784, 762, $626 \mathrm{~cm}^{-1}$. Anal. Calcd for $\mathrm{C}_{15} \mathrm{H}_{22} \mathrm{O}_{2}$ : C, 76.88; H, 9.46; Found: C, 76.67; H, 9.06. 


\section{Microbiology}

\section{Preparation of microorganisms}

A total of 12 microbial cultures belonging to ten bacterial and two fungal species were used in this study (Table 1). The cultures were grown in Mueller-Hinton Broth (Merck) for all the bacterial strains by $24 \mathrm{~h}$ of incubation at $36{ }^{\circ} \mathrm{C}$. C. albicans and C. utilis were grown in Sabouraud Dextrose Broth (Merck) by incubation for $24 \mathrm{~h}$ at $25{ }^{\circ} \mathrm{C}$.

\section{Disc-diffusion assay}

Antimicrobial tests were carried out by disc-diffusion method ${ }^{21-22}$ using $100 \mu \mathrm{L}$ of suspension containing $10^{8} \mathrm{CFU} / \mathrm{mL}$ of bacteria and $10^{6} \mathrm{CFU} / \mathrm{mL}$ of yeast spread on Nutrient Agar (NA), Sabouraud Dextrose Agar (SDA) and Potato Dextrose Agar (PDA) medium, respectively. The blank discs (Oxoid $=6 \mathrm{~mm}$ in diameter) were impregnated with $20 \mu \mathrm{L}$ of the each substances $(105 \mu \mathrm{g} /$ disc $)$ and placed on the inoculated agar. Negative controls were prepared using the same solvents (Methanol) employed to dissolve each substances. Sulbactam $(30 \mu \mathrm{g})+$ Cefoperazona $(75 \mu \mathrm{g})(105 \mu \mathrm{g} / \mathrm{disc})$ was used as positive reference standard to determine the sensitivity of a strain of each microbial species tested. The inoculated plates were incubated at $36{ }^{\circ} \mathrm{C}$ for $24 \mathrm{~h}$ for clinical bacterial strains, $48 \mathrm{~h}$ for yeast and $72 \mathrm{~h}$ for fungi strains. Antimicrobial activity was evaluated by measuring the zone of inhibition against the test organisms.

\section{Results and Discussion}

Previously, multi-substituted 4,5-dihydrofuran derivatives 3a-d and 4a-d have been synthesized by de $\mathrm{Mattos}^{20}$ from the $\mathrm{Mn}(\mathrm{OAc})_{3}$ mediated radical cyclizations of cyclohexane-1,3dion (2a), acetylacetone (2b), ethyl acetoacetate (2c), and benzoylacetone (2d) with limonene, respectively. We resynthesized this compounds 3a-d and 4a-d from the $(S)$-limonene (1) using the same method. In addition, we studied $\mathrm{Mn}(\mathrm{OAc})_{3}$ mediated radical cyclizations of dimedon (2e), methyl acetoacetate (2f), ethyl $N, N$-diethyl-3-oxobutanamide (2g) with $(S)$-limonene (1). Radical cyclization reactions were performed in 2:1:3 molar ratio 1,3-dicarbonyl:( $S$ )-limonene: $\mathrm{Mn}(\mathrm{OAc})_{3}$, respectively, under $\mathrm{N}_{2}$ atmosphere, at $80-60{ }^{\circ} \mathrm{C}$, in acetic acid. The results are given in Scheme 1 and Table 1. The reactions of 1,3-dicarbonyl compounds (2a-g) with $(S)$ limonene resulted in the formation of the corresponding cyclization products (3a-g and $\mathbf{4 a - g}$ ) in $57-87 \%$ yield as a 1:1 mixtures of diastereoisomers (determined by ${ }^{13} \mathrm{C}-\mathrm{NMR}$ ). All efforts to separate the diastereomeric mixture consisting of $\mathbf{3 a - g}$ and $\mathbf{4 a - g}$ by column chromatography, crystallization, and distillation failed.

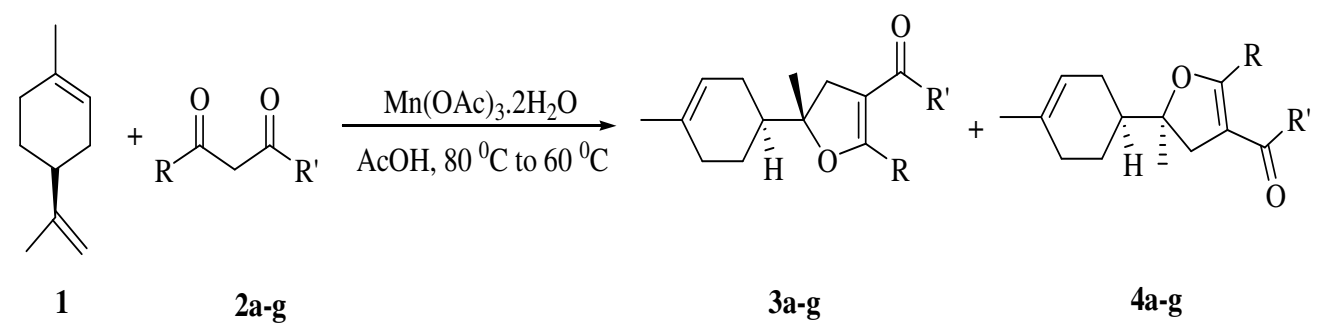

\section{Scheme 1}

The structures of the compounds were determined on the basis of spectroscopic data $\left({ }^{1} \mathrm{H}\right.$ NMR, ${ }^{13} \mathrm{C}$ NMR, IR and Elemental analysis) and comparison with literature data ${ }^{20}$. All spectroscopic data were in accord with the proposed structures. 
Table. 1. Synthesized compounds.

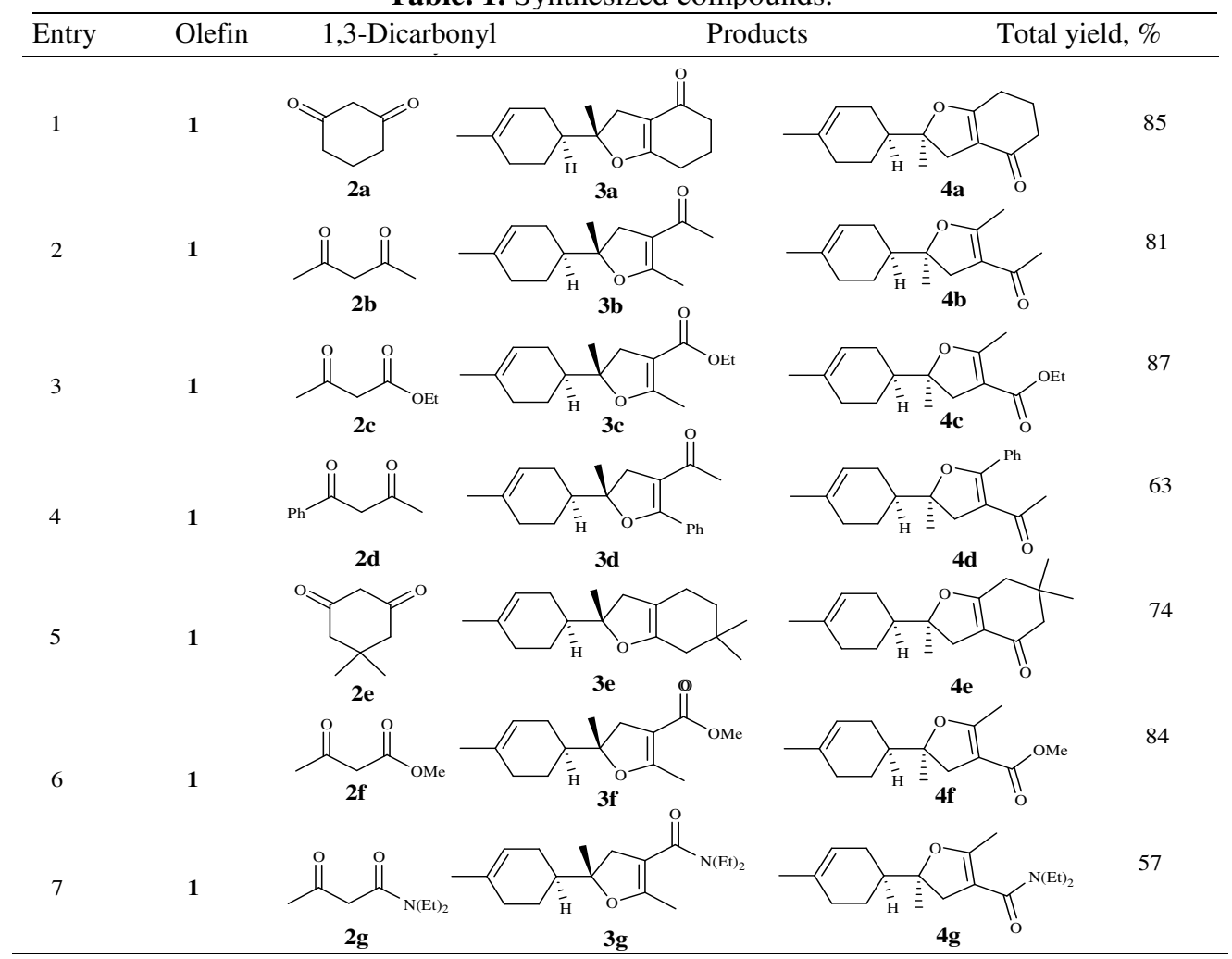

Antibacterial and antifungal activity

The newly synthesized compounds (3a-g and 4a-g) were screened for their antibacterial activity against two yeasts bacteria viz., Candida albicans ATCC 1213, Candida utilis KUEN 1031), five gram positive bacteria viz., Streptococcus homilis, Bacillus cereus DSM 4312, Bacillus subtilis ATCC 6633, Staphylococcus Aureus ATCC 29213, Streptococcus Pyogenez ATCC 176 and five gram negative bacteria viz., Proteus Vulgaris KUEN 1329, Escherichia coli 111, Pseudomonas aeruginosa ATCC 9027, Salmonella enteridis ATCC 13076, Enterococous fecaelis ATCC 29122 by using disc-diffusion method. A reference standard drug SCF (Sulbactam $(30 \mu \mathrm{g})+$ Cefoperazona $(75 \mu \mathrm{g}))$ and methanol were used as positive and negative control, respectively. The experiments were performed in triplicate in order to minimize the errors. Zone of inhibition produced by each compound was measured in $\mathrm{mm}$. The results of antibacterial studies were given in Table 2.

The screening results revealed that the majority of compounds $\mathbf{3 a - g}$ and $\mathbf{4 a - g}$ showed remarkable antimicrobial activity. In particular compound $(\mathbf{3 a}+\mathbf{4 a})$ showed the same activity with positive control (SCF) against the E. fecaelis ATCC 29122. Compound $(\mathbf{3 c}+\mathbf{4 c})$ showed significant activity against $C$. utilis KUEN 1031 and S. homilis. Compound $(\mathbf{3 f}+\mathbf{4 f})$ showed remarkable activity against and $S$ homilis and S enteridis ATCC 13076. Compound (3g + 4g) has shown high potency especially against $C$. utilis KUEN 1031 and E fecaelis ATCC 29122. Compounds $(\mathbf{3 a + 4 a})$ and $(\mathbf{3 b}+\mathbf{4 b})$ have not shown any inhibition against $P$. Vulgaris KUEN 1329, C. utilis KUEN 1031, P. aeruginosa ATCC 9027 and C. albicans ATCC 1213. The authors are indebted to the Department of Chemistry and Biology (Gaziosmapaşa University). 
Table 2. Antimicrobial activity inhibition of diastereomers of $3+4$.

\begin{tabular}{ccccccccccccc}
\hline \multirow{2}{*}{ Compounds s } & \multicolumn{8}{c}{ Microorganisms and inhition zone diameter around test disc, mm } \\
\cline { 2 - 13 } & A & B & C & D & E & F & G & H & I & J & K & L \\
\hline $\mathbf{3 a}+\mathbf{4 a}$ & - & - & 8 & 11 & 14 & - & 13 & 14 & - & 14 & $\mathbf{1 6}$ & - \\
$\mathbf{3 b}+\mathbf{4 b}$ & - & - & 9 & - & 14 & - & 11 & 14 & 8 & 8 & 10 & - \\
$\mathbf{3 c}+\mathbf{4 c}$ & 15 & $\mathbf{1 7}$ & $\mathbf{1 7}$ & 11 & 13 & 14 & 13 & 13 & 13 & 9 & 10 & 14 \\
$\mathbf{3 d}+\mathbf{4 d}$ & 15 & 14 & 15 & 12 & 13 & 8 & 12 & 13 & 12 & 12 & 9 & - \\
$\mathbf{3 e}+\mathbf{4 e}$ & 13 & 14 & 14 & 14 & 15 & - & 12 & 15 & 9 & 10 & 11 & 8 \\
$\mathbf{3 f}+\mathbf{4 f}$ & 16 & 16 & $\mathbf{1 6}$ & 11 & 12 & 16 & 12 & 13 & 15 & 14 & 11 & 16 \\
$\mathbf{3 g}+\mathbf{4 g}$ & 12 & $\mathbf{1 9}$ & 15 & 14 & 13 & 16 & 14 & 10 & 14 & 11 & 13 & 16 \\
$\mathbf{S C F}$ & 22 & 24 & 19 & 22 & 23 & 25 & 26 & 18 & 17 & 17 & 16 & 26 \\
$\mathbf{C H} \mathbf{O H}$ & - & 7 & 7 & 9 & - & 7 & 8 & 19 & 7 & 78 & 8 & 7 \\
\hline
\end{tabular}

SCF: Sulbactam $(30 \mu \mathrm{g})+$ cefoperazona $(75 \mu \mathrm{g})=$ positive control $\mathrm{CH}_{3} \mathrm{OH}$ : Methanol = negative control - : No effect A: Proteus Vulgaris KUEN 1329, B: Candida utilis KUEN 1031, C: Streptococcus homilis, D: E. coli 111, E: Bacillus cereus DSM 4312, F: Pseudomonas aeruginosa ATCC 9027, G: Bacillus subtilis ATCC 6633, H: S. Aureus ATCC 29213, I: Salmonella enteridis ATCC 13076, J: St. Pyogenez ATCC 176, K: Enterococous fecaelis ATCC 29122, L: Candida albicans ATCC 1213.

\section{References}

1. Smith G V, Rihter B, Zsigmond A, Notheisz F and Bartok M, Stud Surf Sci Catal.,1996, 101.

2. Akpolat O, Gündüz G, Özkan F and Beşün N, Appl Catal A: Gen., 2004, 265, 11.

3. Voronkov M V, Kanamarlapudi R C and Richardson P, Tetrahedron Lett., 2005, 46, 6907.

4. Wüst M, Croteau R B, Miyazawa M, Shindo M and Shimada T, Chem Res Toxicol., 2002, 15, 15.

5. Kauffman G S, Harris G D, Dorow R L, B. Stone R P, Jr. Parsons R L, Pesti J A, Magnus N A, Fortunak J M, Confalone P N and Nugent W A, Org Lett.,2000, 2, 3119.

6. Chrisman W, Camara J N, Marcellini K, Singaram B, Goralski C T, Hahsa D L, Rudolf P R, Nicholsonb L W and Borodychukb K K, Tetrahedron Lett., 2001, 42, 5805.

7. Mattos M C S and Bernini R B, J Braz Chem Soc., 2007, 18(5), 1068.

8. Thomas A F and Bessière Y, Nat Prod Rep., 1988, 6, 291.

9. Graebin C S, Eifler-Lima V L and Rosa R G, Catal Commun., 2008, 9(6), 1066.

10. Tokuyasu T, Kunikawa S, Abe M, Masuyama A, Nojima M, Kim H and Wataya SY, J Org Chem., 2003, 68, 7361.

11. Wender P A, Bi F C, Brodney M A and Gosselin F, Org Lett., 2001, 3, 2105.

12. Vuuren S F and Viljoen A M, Flavour Fragr J., 2007, 22, 540.

13. Onitsuka S and Nishino H, Tetrahedron, 2003, 59, 755.

14. Dean F A, Naturally Occuring Oxygen Ring Compounds, Butterworths, London, 1963.

15. Bosshard P, Eugster C H, Katritzky A R and Boulton A J, Eds., Advances in Heterocyclic Chemistry, Academic, New York, 1966, 7, 377-588.

16. Bijan P D and Boykin D W, J Med Chem., 1977, 20, 531.

17. Simpson J I, Lee M, Kumar A, Boykin D W and Neidle S, Bioorg Med Chem Lett., 2000, 10, 2593.

18. Xiao G, Kumar A, Li K, Rigl T, Bajic M, Davis T M, Boykin D W and Wilson W D, Bioorg Med Chem., 2001, 9, 1097.

19. Schwikkard S and Heerden F, Nat Prod Rep., 2002, 19, 675.

20. Mattos M C S, Souza S P L and Elias S M, Heterocycl Commun., 2003, 9(3), 247.

21. Karaman İ, Şahin F, Güllüce $M$, Ögütçü $H$, Şengül $M$ and Adıgüzel $A, J$ Ethnopharmacol., 2003, 85, 231.

22. Murray P P, Baron E J, Pfaller M A, Tenover F C and Yolke R H, Manual of Clinical Microbiology, ASM, Washington, DC. 1995, 6. 


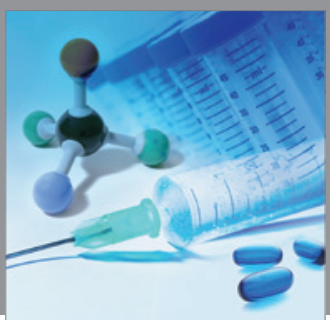

International Journal of

Medicinal Chemistry

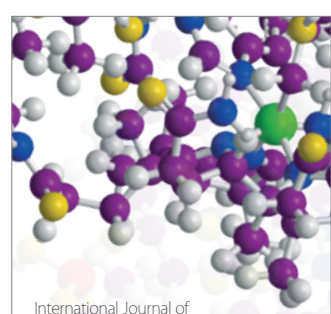

Carbohydrate Chemistry

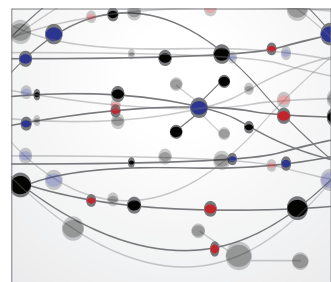

The Scientific World Journal
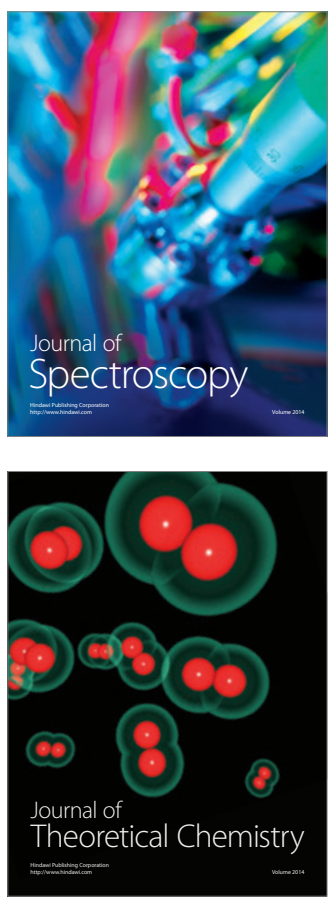
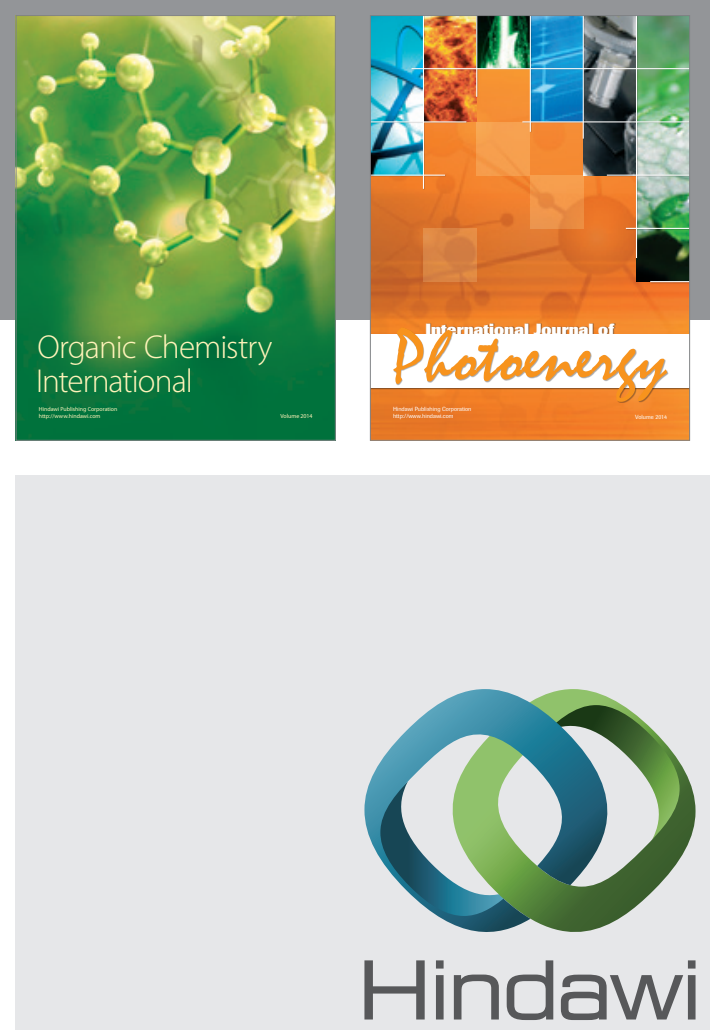

Submit your manuscripts at

http://www.hindawi.com
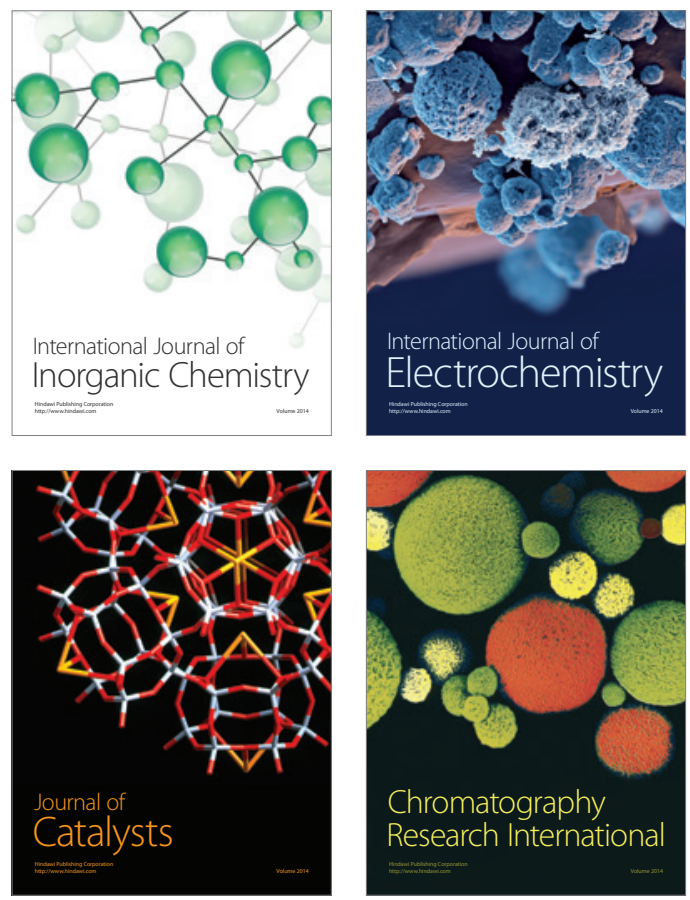
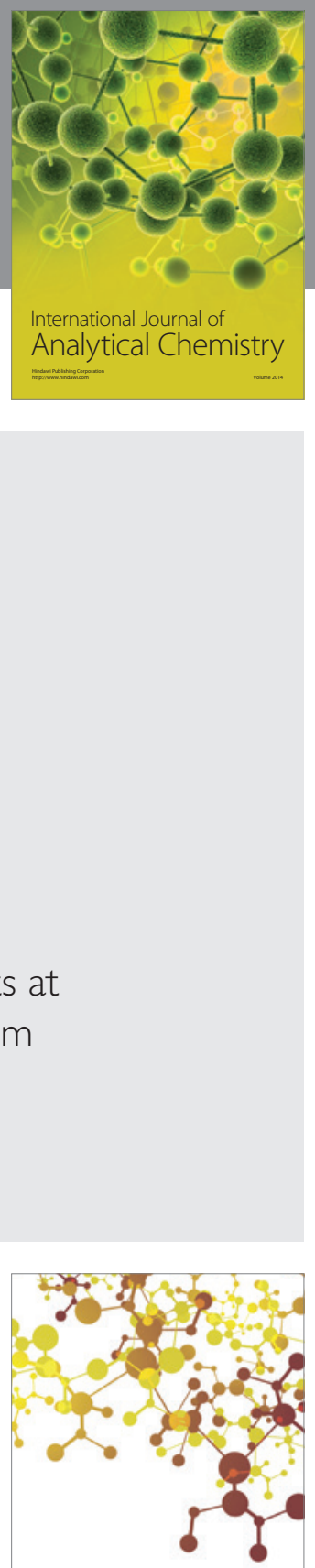

Journal of

Applied Chemistry
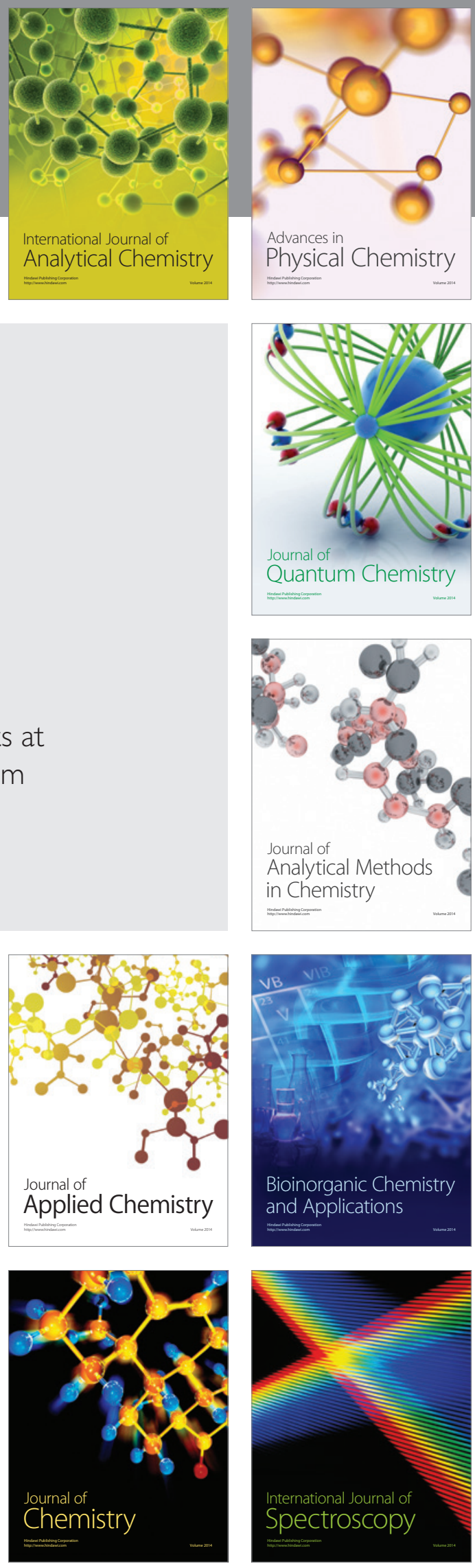